\title{
Long-Acting GnRH Agonist Improves IVF Outcomes of Young Patients with Diminished Ovarian Reserve by Increasing Endometrial Receptivity.
}

xuemei Liu ( $\square$ xuemeiliu02@yeah.net )

Qindao University Medical College Affiliated Yantai Yuhuangding Hospital https://orcid.org/00000001-9636-1625

\section{Hongchu Bao}

Qindao University Medical College Affiliated Yantai Yuhuangding Hospital

\section{Qinglan Qu}

Qindao University Medical College Affiliated Yantai Yuhuangding Hospital

\section{Zhenyu Guo}

Qindao University Medical College Affiliated Yantai Yuhuangding Hospital

\section{Wenshu Li}

Qindao University Medical College Affiliated Yantai Yuhuangding Hospital

\section{Huishan Zhao}

Qindao University Medical College Affiliated Yantai Yuhuangding Hospital

\section{Research}

Keywords: Diminished ovarian reserve, In vitro fertilization, long-acting GnRH agonist, clinical pregnancy rate, implantation rate

Posted Date: July 1st, 2020

DOI: https://doi.org/10.21203/rs.3.rs-36728/v1

License: (c) (7) This work is licensed under a Creative Commons Attribution 4.0 International License. Read Full License 


\section{Abstract}

Background: Up to now, there is not sufficient evidence to recommend a treatment optimizing in vitro fertilization (IVF) outcomes for diminished ovarian reserve (DOR). This study is aimed to investigate whether long-acting gonadotrophin-releasing hormone $(\mathrm{GnRH})$ agonist long protocol in follicular phase could improve the IVF cycle outcomes for young patients with DOR when compared with GnRH antagonist and mild ovarian stimulation protocols.

Methods: This retrospective cohort study was carried out from June 2015 to March 2019. 338 patients aged 20-40 years with DOR who underwent first IVF between were enrolled. These patients were assigned to three groups depending on the ovarian stimulation protocols. The outcome parameters of IVF were compared in each group. The demographic and reproductive characteristics were calculated by MannWhitney, Kruskal-Wallis or chi-square test as appropriate. We evaluated the clinical outcomes of IVF cycle between the three groups using univariate and multivariate logistic regression analyses. In addition, we evaluated the morphology and coverage of pinopode and expression of HOXA10 in endometrium during implantation window between three groups by scanning electron microscope and qRT-PCR.

Results: Patients who received long-acting GnRH agonist long protocol had significantly higher clinical pregnancy rates $(66.67 \%, 42.17 \%$ and $39.02 \%$, respectively; $P=.010$ and .005$)$, implantation rates $(46.15 \%$, $29.71 \%$, and $28.57 \%$, respectively; $P=.041$ and .025$)$ and ongoing pregnancy rates $(60.00 \%, 34.94 \%$, and $36.59 \%$, respectively; $P=.018$ and .004 ). They also had significantly higher duration of stimulation, total dose of gonadotrophins and endometrial thickness than the other two groups (P凶.001). However, serum luteinizing hormone $(\mathrm{LH})$ and estradiol $\left(\mathrm{E}_{2}\right)$ level on gonadotrophins initiation day, serum LH level on human chorionic gonadotropin (hCG) day, the embryos transferred cancellation rate and abnormal endometrium rate were significantly lower among women who received long-acting $\mathrm{GnRH}$ agonist $(P$ ه.001). In addition, we found that long-acting GnRH agonist could improve the development of pinopode and mRNA of HOXA10 (Pख.05).

Conclusions: To our knowledge, this is the first time that the benefit of long-acting GnRH agonist long protocol in follicular phase in young DOR patients has been reported. Though this novel protocol may have further suppressed response, it can increase endometrial receptivity, reduce cycle cancellation rate and improve IVF cycle clinical outcomes for these patients compared with mild stimulation and $\mathrm{GnRH}$ antagonist protocols.

\section{Plain English Summary}

Diminished ovarian reserve (DOR) is a major challenge in the treatment of infertility. There are no treatments available to improve IVF outcomes for DOR. In this study long-acting GnRH agonist should improve IVF outcomes of young patients with DOR.

338 patients aged $20-40$ years with DOR who underwent first IVF were enrolled. They were assigned to three groups depending on the ovarian stimulation protocols, including long-acting GnRH agonist long 
protocol in follicular phase, mild ovarian stimulation and $\mathrm{GnRH}$ antagonist protocol. The outcome parameters of IVF and markers of endometrial receptivity were compared in each group.

Long-acting GnRH agonist could significantly increase clinical pregnancy rates, implantation rates and ongoing pregnancy rates. In addition, we found that long-acting $\mathrm{GnRH}$ agonist could improve endometrial thickness and the development of pinopode and mRNA of HOXA10.

In conclusion, long-acting GnRH agonist long protocol in follicular phase could improve IVF cycle clinical outcomes by increasing endometrial receptivity for young DOR patients.

\section{Background}

Ovarian reserve refers to the number and quality of follicles in the ovary. It can indicate the women's reproductive potential and plays an important role in fertility in reproductive age women. Diminished ovarian reserve (DOR) refers to a reduced number of ovarian follicles, causing poor fertility outcomes [1]. The clinical management of patients with DOR remains a major challenge in the treatment of infertility. Recently, the incidence of DOR is growing, approximately $10 \%$ among infertile women [2-3]. DOR not only refers to the reduced number and quality of remaining oocytes in the ovary, but also contributes to poor ovarian response (POR), increased cycle cancellation and miscarriage rate, decreased clinical pregnancy and live birth rate after treatment with assisted reproduction technology (ART) [4-6].

To attempt to optimize in vitro fertilization (IVF) outcomes for DOR, a lot of treatments have been proposed, such as different ovarian stimulation protocols, luteal phase pretreatments and various supplements and modifications during ovarian stimulation [7-11]. However, most of the therapeutic strategies had limited success. Up to now, there is not sufficient evidence to recommend a treatment optimizing IVF outcomes for DOR [12].

Ovarian simulation is one important step of IVF cycle. Therefore, it is crucial to select an optimized ovarian stimulation protocol for patients with DOR. But, it is very difficult for such patients, since few oocytes are retrieved in a single ovarian stimulation cycle $[8,11]$. Considering the few antral follicle count (AFC) of DOR patients, the conventional ovarian stimulation protocols are mainly mild ovarian stimulation and gonadotrophin-releasing hormone $(\mathrm{GnRH})$ antagonist protocols $[7,8,10,13]$. However, the clinical pregnancy rate has been very low.

Recently, long-acting GnRH agonist long protocol in follicular phase is proposed in controlled ovarian stimulation of IVF cycle [14-19]. During this protocol, a long-acting GnRH agonist triptorelin is given as a single dose for pituitary desensitization on day 2-3 of menstrual cycle. Some studies have shown that this protocol could significantly improve endometrial receptivity and clinical outcomes compared with other protocols [16-19].

As we all know, long-term pituitary down-regulation before IVF could improve pregnancy outcomes in women with endometriosis $[14,15,20,21]$. Therefore, in our center, long-acting GnRH agonist protocol in 
follicular phase was primarily used for patients with endometriosis. We found that these endometriosis patients in addition to DOR have excellent clinical pregnancy using this protocol. We believe that this protocol may be beneficial to patients with DOR. Hence, long-acting GnRH agonist long protocol in follicular phase is administrated for young patients with DOR in our center. However, the role of this protocol is still unclear for these patients. To explore this problem, for the first time, we set up a retrospective cross-sectional study to evaluate the effect of long-acting $\mathrm{GnRH}$ agonist long protocol in follicular phase on IVF clinical outcomes of young patients with DOR compared with conventional protocols.

\section{Methods}

\section{Trial design and participants' characteristics}

This was designed as a retrospective cohort study. We have retrospectively reviewed the clinical data of all young patients with DOR, who underwent their first in vitro fertilization (IVF) cycle at Yantai Yuhuangding Hospital from June 1st 2015 to March 31st 2019. The study protocol was approved by the Ethical Committee of Yantai Yuhuangding Hospital. The study conformed to the "Declaration of Helsinki for Medical Research involving Human Subjects". Patients' characteristics and cycle parameters were obtained from patient medical records.

It included young patients age $<40$ years with DOR eligible for IVF. Currently, there is no uniform definition for DOR. In this study, the definition of DOR is as following : (i) woman with any of the risk factors for POR and/or (ii) an abnormal ovarian reserve test (i.e., AFC $<7$ follicles or anti mullerian hormone (AMH) $<1.2 \mathrm{ng} / \mathrm{ml})[12,22]$. Exclusion criteria included patients with severe uterine malformation, severe uterine adhesions, chromosomal abnormality, hydrosalpinx (if not surgically removed or ligated), antiphospholipid syndrome, thyroid or adrenal dysfunction, and severe impairment of renal or hepatic function.

\section{Procedure of IVF}

As shown in Fig.1, all patients undergoing IVF cycles received long-acting GnRH agonist long protocol in follicular phase, mild ovarian stimulation or $\mathrm{GnRH}$ antagonist protocol. In long-acting $\mathrm{GnRH}$ agonist long protocol in follicular phase, a long-acting GnRHa triptorelin acetate $(3.75 \mathrm{mg}, 1.88 \mathrm{mg}$ or $1.25 \mathrm{mg}$ ) was used as a single dose to make pituitary desensitization on day 2-3 of menstrual cycle if the vaginal Bscan ultrasonography shows no cysts and follicles $\triangle 10 \mathrm{~mm}$. 28 days after the injection, ultrasound scan, serum estradiol $\left(\mathrm{E}_{2}\right)$, luteinizing hormone $(\mathrm{LH})$, follicle-stimulating hormone $(\mathrm{FSH})$ and progesterone $(\mathrm{P})$ were examined to evaluate pituitary down-regulation. If it reaches the down-regulation criteria (follicle diameter $₫ 5 \mathrm{~mm}, \mathrm{E}_{2} \varangle 50 \mathrm{pg} / \mathrm{mL}$, LH $₫ 5 \mathrm{IU} / \mathrm{L}$, endometrium $₫ 5 \mathrm{~mm}$ ), gonadotrophins will be started according to the patients' age, AMH, body mass index (BMI), AFC and medical history.

In GnRH antagonist protocol, gonadotropins were used on the third day of the menstrual cycle. GnRH antagonist ganirelix was used when the dominant follicle reached $12 \mathrm{~mm}$ in diameter, $\mathrm{E}_{2}$ concentrations 


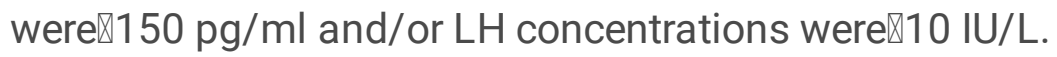

In mild ovarian stimulation protocol, clomiphene and human menopausal gonadotropin (HMG) were administered from the third day of the menstrual cycle to the day of human chorionic gonadotropin (hCG) trigger injection.

During gonadotrophin stimulation, $\mathrm{E}_{2}, \mathrm{P}, \mathrm{LH}$, follicle size measurements, and endometrial thickness were monitored until the day of hCG trigger injection. When one leading follicle reached a mean diameter of 18 $\mathrm{mm}$ or two leading follicles reached $17 \mathrm{~mm}$, hCG was given to trigger ovulation. After 34-36 h, oocytes were retrieved transvaginally. Conventional insemination was performed as indicated. Ultrasound-guided fresh embryo transfer was performed on the third day after oocyte retrieval. The number of embryos transferred was one or two depending on the number of available embryos. The excess viable embryos were cryopreserved or cultured to blastocyst stage and then cryopreserved for subsequent frozen embryo transfer (FET) cycles. Fresh embryo transfer was cancelled if the embryo and endometrium were not synchronous or women had some factors seriously affecting embryo implantation. Cleavage stage embryos were graded using a standardized system [23]. The high-quality embryos included embryos of grade 1 or 2 . The luteal phase was supported from the day of oocyte retrieval. $200 \mathrm{mg}$ progesterone (Utrogest $^{\text {TM }} 200$, Besins-Iscovesco, France) were vaginal administered three times daily until the early pregnancy test. A quantitative early pregnancy test was performed on the 14th day after embryo transfer. Clinical pregnancy was confirmed if the fetal heartbeat was observed by transvaginal ultrasound after 34 days from embryo transfer. Ongoing pregnancy was defined as a live fetus on ultrasound beyond three months of gestation.

\section{Study design}

All patients were assigned to three groups depending on the ovarian stimulation protocols. The demographic and reproductive characteristics were calculated by descriptive statistics. We evaluated the clinical outcomes of IVF cycle while adjusting for potential confounding factors between the three groups. Endometrial tissues were obtained on Day 5 after oocyte recovery from the three group infertile women with tubal factor. Then we evaluated the morphology and coverage of pinopode and expression of HOXA10 between three groups.

\section{Endometrial tissue}

Endometrial tissues were collected from 24 women under their written informed consent following the protocol approved by the Institutional Review Board of Yantai Yuhuangding Hospital. The samples were obtained on Day 5 after oocyte recovery from women who rejected fresh embryo transfer because of personal reasons. Eight cases were selected in each group. The tissue sample of each woman was divided into two parts. One part was kept frozen at $-80^{\circ} \mathrm{C}$ for subsequent qRT-PCR. The other was fixed with $2.5 \%$ glutaraldehyde for scanning electron microscope analysis.

\section{Scanning electron microscopy}


The morphology and coverage of pinopode in endometrium was evaluated by scanning election microscopy. The endometrial samples were fixed in $2.5 \%$ glutaraldehyde for 4 hours. Followed by rinsing three times in $0.1 \mathrm{~mol} / \mathrm{l}$ phosphate-buffered saline (PBS) for 10 minutes, the samples were dehydrated in graded alcohols and then dried in a critical point drier. Subsequently, samples were coated with palladium gold and examined by a scanning electron microscope. Pinopode development and coverage scoring was assessed as previously described.

\section{qRT-PCR of mRNA expression}

Total RNA was extracted from endometrial tissues with Trizol reagent (Invitrogen, Carlsbad, CA) and reversely transcribed using QuantiTect Reverse Transcription Kit (Qiagen GmbH, Hilden, Germany) following the manufacturer's instructions. QuantiNova SYBR Green PCR Kit was used to detect the HOXA10 mRNA expression. The primers were listed as follows: HOXA10 forward, 5'GCCCTTCCGAGAGCAGCAAAG 3' and reverse, 5' AGGTGGACGCTGCGGCTAATCTCTA3'; $\beta$-actin forward, 5'-CTGGACTTCGAGCAAGAGATG-3' and reverse, 5'-GAGTTGAAGGTAGTTTCGTGGA-3'. The reaction was performed as bellow: $95^{\circ} \mathrm{C}$ for 2 minutes, followed by 40 cycles of $95^{\circ} \mathrm{C}$ for 5 seconds and $60^{\circ} \mathrm{C}$ for 10 seconds.

\section{Main outcome measures}

The primary outcome was clinical pregnancy rate. Secondary outcomes were implantation rate, ongoing pregnancy rate, high quality embryo rate, blastocyst formation rate, endometrial thickness on HCG day, abnormal endometrium rate, embryo transfer cancellation rate, serum LH level on gonadotrophins initiation and HCG day, serum $E_{2}$ level on gonadotrophins initiation and HCG day, duration of stimulation and total dose of gonadotrophins.

\section{Statistical methods}

Descriptive statistics were carried out to describe the baseline characteristics of the patients. Continuous variables with normal distribution were expressed as mean \pm standard deviation (SD), non-normal continuous variables as median (interquartile range, IQR). Categorical variables were presented in terms of frequency and percentages. The Mann-Whitney $U$ test or Kruskal-Wallis test was performed to compare continuous variables, followed by a Dunn-Bonferroni test for post hoc comparisons. The chi-square test was employed to compare the difference of categorical variables, and the Bonferroni-corrected P-value was used for multiple comparisons. Univariate and multivariate Logistic regression models were performed to generate crude and adjusted Odd ratio (OR) for exploring the effects of different treatment on clinical outcomes. The selection of variables was performed using a backward stepwise process with the Akaike information criterion.

All statistical tests were performed using R software (version 3.5.2, http://www.r-project.org/). All tests were two-sided, and $\mathrm{P}<0.05$ was considered statistically significant. 


\section{Results}

The process of patient selection is shown in Fig. 2. In this study, 338 patients were included. 180 patients received the mild ovarian stimulation protocol (group 1), 122 received $\mathrm{GnRH}$ antagonist protocol (group 2), and 36 received long-acting GnRH agonist long protocol in follicular phase (group 3).

Patients' characteristics and demographics are presented in Table 1, as no statistical differences were observed between the groups regarding age, $\mathrm{BMI}$, the duration of infertility and previous pregnancies ( $P \otimes .05)$. The infertility diagnosis, AMH and AFC were significantly different between groups ( $P \otimes .001, P$ $=.033$ and $P=.011$, respectively). The incidence of endometriosis was significantly higher in group 3 when compared with groups 1 and 2. AFC was significantly higher in group 3 than group 1 . In addition, higher $\mathrm{AMH}$ was noted in group 2 when compared with group 1. 
Table 1

Patients baseline characteristics between the three groups.

\begin{tabular}{|c|c|c|c|c|}
\hline Variables & Group1 & Group2 & Group 3 & $P$ value \\
\hline Number of cycles & 180 & 122 & 36 & \\
\hline Age(y) & $34(31-36)$ & $34(31-36)$ & $33.5(29.5-36)$ & .793 \\
\hline Duration of infertility(y) & $4(2-6)$ & $3(2-5)$ & $4(2-8)$ & .458 \\
\hline $\mathrm{BMI}\left(\mathrm{kg} / \mathrm{m}^{2}\right)$ & $23.0(21.3-25.4)$ & $22.6(20.3-24.8)$ & $\begin{array}{l}22.3(20.3- \\
25.3)\end{array}$ & .252 \\
\hline $\mathrm{AMH}(\mathrm{ng} / \mathrm{ml})$ & $\begin{array}{l}0.97(0.50- \\
1.36)^{\mathrm{a}}\end{array}$ & $\begin{array}{l}1.15(0.82- \\
1.36)^{\mathrm{a}}\end{array}$ & $\begin{array}{l}1.10(0.72- \\
1.49)\end{array}$ & .033 \\
\hline $\mathrm{AFC}(\mathrm{n})$ & $6(4-8)^{b}$ & $6(5-8)$ & $7(6-9)^{b}$ & .011 \\
\hline Previous pregnancy & & & & .367 \\
\hline NO $(n, \%)$ & $92(51.1 \%)$ & $53(43.4 \%)$ & $19(52.8 \%)$ & \\
\hline Yes $(n, \%)$ & $88(48.9 \%)$ & $69(56.6 \%)$ & $17(47.2 \%)$ & \\
\hline $\begin{array}{l}\text { Diagnosis in addition to } \\
\text { DOR }\end{array}$ & & & & $<.001$ \\
\hline Male factor $(n, \%)$ & $19(10.6 \%)$ & $10(8.2 \%)$ & $1(2.8 \%)$ & \\
\hline Tubal factor (n, \%) & $84(46.7 \%)$ & $64(52.5 \%)$ & $9(25.0 \%)$ & \\
\hline Mixed $(n, \%)$ & $28(15.6 \%)$ & $17(13.9 \%)$ & $2(5.6 \%)$ & \\
\hline Endometriosis (n, \%) & $20(11.1 \%)$ & $13(10.7 \%)$ & $20(55.6 \%)$ & \\
\hline Unexplained (n, \%) & $29(16.1 \%)$ & $18(14.8 \%)$ & $4(11.1 \%)$ & \\
\hline \multicolumn{5}{|c|}{ Note: *: Values are significantly different between groups $(P<0.05)$. } \\
\hline \multicolumn{5}{|c|}{ a Group 1 vs. group 2, Bonferroni-corrected $\mathrm{P}=.04$. } \\
\hline b Group 1 vs. group 3, B & corrected $P=0$ & & & \\
\hline
\end{tabular}

Table 2 presents the ovarian stimulation characteristics and embryology outcomes of IVF between the three groups. Serum $P$ and $E_{2}$ levels on HCG day, normal fertilization rate, high quality embryo rate, blastocyst formation rate, number of embryos transferred, no oocyte retrieved rate and no available embryos rate were similar ( $P \bigotimes$.05). Serum LH and $\mathrm{E}_{2}$ level on gonadotrophins initiation day, serum LH level on HCG day, embryo transfer cancellation rate and abnormal endometrium rate (including thin and asynchronous endometrium with embryo) were significantly lower among women who received longacting GnRH agonist long protocol than the other two groups (PX.001). However, the duration of stimulation, amount of gonadotrophins and endometrial thickness were significantly higher in group of 
long-acting $\mathrm{GnRH}$ agonist compared with the other two groups ( $P \Downarrow .001)$. The number of oocytes retrieval, mature oocytes and high quality embryos transferred were significantly higher in group of long-acting $\mathrm{GnRH}$ agonist only than the group of mild stimulation protocol $(P \otimes .001, \bigotimes .001$ and $P=.027)$. 
Table 2

Ovarian stimulation parameters and embryology outcomes between the three groups.

\begin{tabular}{|c|c|c|c|c|}
\hline Variables & Group1 & Group2 & Group 3 & $\begin{array}{l}P \\
\text { value }\end{array}$ \\
\hline Number of cycles & 180 & 122 & 36 & \\
\hline $\begin{array}{l}\text { LH on Gn initiation day } \\
(\mathrm{U} / \mathrm{L})\end{array}$ & $4.30(3.30-5.67)^{\mathrm{a}}$ & $4.64(3.45-6.20)^{b}$ & $\begin{array}{l}0.57(0.32- \\
0.79)^{\mathrm{a}, \mathrm{b}}\end{array}$ & $<.001$ \\
\hline $\begin{array}{l}\mathrm{E}_{2} \text { on } \mathrm{Gn} \text { initiation day } \\
(\mathrm{ng} / \mathrm{ml})\end{array}$ & $\begin{array}{l}33.28(22.02- \\
44.86)^{\mathrm{a}}\end{array}$ & $\begin{array}{l}32.97(22.19- \\
46.55)^{b}\end{array}$ & $\begin{array}{l}5.32(5.00- \\
13.92)^{a, b}\end{array}$ & $<.001$ \\
\hline Duration of stimulation (d) & $8(6-9)^{a}$ & $8(7-9)^{b}$ & $11(9-12)^{a, b}$ & $<.001$ \\
\hline The amount of Gn (IU) & $\begin{array}{l}1350(1050- \\
1800)^{\mathrm{a}}\end{array}$ & $\begin{array}{l}1875(1519- \\
2256)^{b}\end{array}$ & $\begin{array}{l}2775(2250- \\
3413)^{a, b}\end{array}$ & $<.001$ \\
\hline $\begin{array}{l}\text { EM thickness on HCG day } \\
(\mathrm{mm})\end{array}$ & $7(6-9)^{a}$ & $10(8-11)^{b}$ & $11(9-12)^{a, b}$ & $<.001$ \\
\hline P on HCG day $(\mathrm{ng} / \mathrm{ml})$ & $0.70(0.47-1.00)$ & $0.68(0.45-0.93)$ & $0.62(0.42-0.92)$ & .595 \\
\hline LH on HCG day (U/L) & $\begin{array}{l}7.47(4.93- \\
10.74)^{\mathrm{a}}\end{array}$ & $3.29(2.42-5.41)^{b}$ & $\begin{array}{l}0.75(0.28- \\
1.24)^{\mathrm{a}, \mathrm{b}}\end{array}$ & $<.001$ \\
\hline $\mathrm{E}_{2}$ on HCG day $(\mathrm{pg} / \mathrm{ml})$ & $\begin{array}{l}998.8(654.0- \\
1472.5)\end{array}$ & $\begin{array}{l}1010.5(679.7- \\
1390.3)\end{array}$ & $\begin{array}{l}892.4(474.7- \\
1557.3)\end{array}$ & .691 \\
\hline Oocytes retrieved (n) & $3(2-4)^{a, c}$ & $3(2-4)^{c}$ & $4(3-5)^{a}$ & $<.001$ \\
\hline Mature oocytes (n) & $2(1-3)^{a}$ & $3(2-3)$ & $3(2-5)^{a}$ & $<.001$ \\
\hline $\begin{array}{l}\text { Normal fertilization rate } \\
(\%)\end{array}$ & $75.43 \%(353 / 468)$ & $71.47 \%(278 / 389)$ & $76.29 \%(103 / 135)$ & .339 \\
\hline $\begin{array}{l}\text { High quality embryo rate } \\
(\%)\end{array}$ & $69.06 \%(221 / 320)$ & $65.81 \%(179 / 272)$ & $63.37 \%(64 / 101)$ & .499 \\
\hline
\end{tabular}

Note: *: Values are significantly different between groups $(P<0.05)$.

EM: endometrial; $\mathrm{HQ}$ : high quality; Gn: gonadotrophins; $\mathrm{ET}$ : embryo transfer.

Abnormal endometrium includes thin endometrum and asynchronous endometirum with embryo.

a Group 1 vs. group 3, Bonferroni-corrected $\mathrm{P}<.001$.

${ }^{\mathrm{b}}$ Group 2 vs. group 3, Bonferroni-corrected $\mathrm{P}<.001$.

${ }^{\mathrm{c}}$ Group 1 vs. group 2, Bonferroni-corrected $\mathrm{P}=.004$.

${ }^{d}$ Group 1 vs. group 3, Bonferroni-corrected $\mathrm{P}=.024$. 


\begin{tabular}{|lllll|}
\hline Variables & Group1 & Group2 & Group 3 & $\begin{array}{c}\text { P } \\
\text { value }\end{array}$ \\
\hline $\begin{array}{l}\text { Blastocyst formation rate } \\
(\%)\end{array}$ & $42.86 \%(3 / 7)$ & $46.25 \%(37 / 80)$ & $51.16 \%(22 / 43)$ & .875 \\
\hline Embryos transferred (n) & $2(1-2)$ & $2(1-2)$ & $2(1-2)$ & .201 \\
\hline HQ embryos transferred(n) & $1(1-2)^{\mathrm{d}}$ & $1(1-2)$ & $2(1-3)^{\mathrm{d}}$ & .027 \\
\hline $\begin{array}{l}\text { No oocyte retrieved rate } \\
(\%)\end{array}$ & $2.78 \%(5 / 180)$ & $0.82 \%(1 / 122)$ & $5.56 \%(2 / 36)$ & .152 \\
\hline $\begin{array}{l}\text { No available embryos rate } \\
(\%)\end{array}$ & $5.00 \%(9 / 180)$ & $2.46 \%(3 / 122)$ & $8.33 \%(3 / 36)$ & .220 \\
\hline $\begin{array}{l}\text { Abnormal endometrium } \\
\text { rate (\%) }\end{array}$ & $\begin{array}{l}55.56 \% \\
(100 / 180)^{\mathrm{a}}\end{array}$ & $15.57 \%(19 / 122)^{\mathrm{b}}$ & $2.78 \%(1 / 36)^{\mathrm{a}, \mathrm{b}}$ & $<.001$ \\
\hline ET cancellation rate $(\%)$ & $\begin{array}{l}77.22 \% \\
(139 / 180)^{\mathrm{a}}\end{array}$ & $31.97 \%(39 / 122)^{\mathrm{b}}$ & $16.67 \%(6 / 36)^{\mathrm{a}, \mathrm{b}}$ & $<.001$ \\
\hline Note: *: Values are significantly different between groups $(\mathrm{P}<0.05)$. & & \\
\hline EM: endometrial; HQ: high quality; Gn: gonadotrophins; ET: embryo transfer. & \\
\hline Abnormal endometrium includes thin endometrum and asynchronous endometirum with embryo. \\
\hline a Group 1 vs. group 3, Bonferroni-corrected $\mathrm{P}<.001$.
\end{tabular}

These results indicate that long-acting GnRH agonist long protocol may further suppress ovarian response, lengthen the time of ovarian stimulation, improve the endometrial thickness on HCG day, and reduce embryo transfer cancellation rate and abnormal endometrium rate as compared with the other two groups.

Of the 338 fresh cycles with oocyte retrieval, 154 cycles had fresh embryo transfer. Table 3 depicts the patient and IVF cycle characteristics by pregnancy outcome. Ongoing pregnancy was associated with normal fertilization rate $(P=.009)$, the number of oocytes retrieved $(P \otimes .001)$, embryos transferred $(P$ $=.001)$, and high quality embryos transferred $(P=.004)$. AFC and endometrial thickness were greater, while serum LH level on gonadotrophins initiation and HCG day were lower in the ongoing pregnancy group. However, they did not achieve statistical significance. In addition, $\operatorname{AFC}(P=.037)$, serum $P$ level on HCG day $(P=.041)$, high quality embryo rate $(P=.047)$, the number of embryo transferred $(P \otimes .001)$ and high quality embryos transferred $(P=.001)$ were significantly associated with clinical pregnancy. The 
endometrial thickness was higher, while the duration of infertility and serum LH level on gonadotrophins initiation and HCG day were lower in the clinical pregnancy group. However, they did not achieve statistical significance. 
Table 3

Patients and IVF cycle characteristics by pregnancy outcome

\begin{tabular}{|c|c|c|c|c|c|c|}
\hline Variables & Pregnant & Not pregnant & $\begin{array}{l}P \\
\text { value }\end{array}$ & $\begin{array}{l}\text { Ongoing } \\
\text { pregnancy }\end{array}$ & $\begin{array}{l}\text { No ongoing } \\
\text { pregnancy }\end{array}$ & $\begin{array}{l}P \\
\text { value }\end{array}$ \\
\hline $\begin{array}{l}\text { Number of } \\
\text { cycles }\end{array}$ & 71 & 83 & & 62 & 92 & \\
\hline Age(y) & $34(30-35)$ & $35(31-37)$ & .199 & $34(30-35)$ & $34(31-37)$ & .291 \\
\hline $\begin{array}{l}\text { Duration of } \\
\text { infertility }(y)\end{array}$ & $3(2-5)$ & $4(2-7)$ & .056 & $3.5(2.0-5.0)$ & $3.3(2.0-6.8)$ & .378 \\
\hline $\mathrm{BMI}\left(\mathrm{kg} / \mathrm{m}^{2}\right)$ & $\begin{array}{l}22.7(20.6- \\
25.1)\end{array}$ & $\begin{array}{l}22.8(20.5- \\
25.4)\end{array}$ & .889 & $\begin{array}{l}22.8(20.8- \\
25.3)\end{array}$ & $\begin{array}{l}22.7(20.4- \\
25.4)\end{array}$ & .922 \\
\hline $\mathrm{AMH}(\mathrm{ng} / \mathrm{ml})$ & $\begin{array}{l}1.10(0.73- \\
1.46)\end{array}$ & $\begin{array}{l}1.11(0.93- \\
1.35)\end{array}$ & .921 & $\begin{array}{l}1.07(0.70- \\
1.46)\end{array}$ & $\begin{array}{l}1.11(0.90- \\
1.35)\end{array}$ & .963 \\
\hline $\mathrm{AFC}(\mathrm{n})$ & $7(5-8)$ & $6(5-8)$ & $.037^{*}$ & $7(5-8)$ & $6(5-8)$ & .208 \\
\hline $\begin{array}{l}\text { Previous } \\
\text { pregnancy }\end{array}$ & & & 1 & & & .718 \\
\hline NO $(n, \%)$ & $35(50 \%)$ & $42(50 \%)$ & & $29(46.8 \%)$ & $47(51.1 \%)$ & \\
\hline Yes (n, \%) & $35(50 \%)$ & $42(50 \%)$ & & $33(53.2 \%)$ & $45(48.9 \%)$ & \\
\hline $\begin{array}{l}\text { Diagnosis in } \\
\text { addition to } \\
\text { DOR }\end{array}$ & & & .228 & & & .517 \\
\hline $\begin{array}{l}\text { Male factor } \\
(n, \%)\end{array}$ & $6(8.6 \%)$ & $6(7.1 \%)$ & & $6(9.7 \%)$ & $6(6.5 \%)$ & \\
\hline $\begin{array}{l}\text { Tubal factor } \\
(n, \%)\end{array}$ & $18(25.7 \%)$ & $12(14.3 \%)$ & & $25(40.3 \%)$ & $47(51.1 \%)$ & \\
\hline Mixed (n, \%) & $12(17.1 \%)$ & 11(13.1\%) & & $8(12.9 \%)$ & $15(16.3 \%)$ & \\
\hline $\begin{array}{l}\text { Endometriosis } \\
(\mathrm{n}, \%)\end{array}$ & 26(37.1\%) & $46(54.8 \%)$ & & $15(24.2 \%)$ & $15(16.3 \%)$ & \\
\hline $\begin{array}{l}\text { Unexplained } \\
(\mathrm{n}, \%)\end{array}$ & $8(11.4 \%)$ & $9(10.7 \%)$ & & $8(12.9 \%)$ & $9(9.8 \%)$ & \\
\hline $\begin{array}{l}\mathrm{LH} \text { on } \mathrm{Gn} \\
\text { initiation day } \\
(\mathrm{U} / \mathrm{L})\end{array}$ & $\begin{array}{l}3.54(0.94- \\
5.21)\end{array}$ & $\begin{array}{l}4.19(2.97- \\
5.85)\end{array}$ & .055 & $\begin{array}{l}3.70(0.94- \\
5.54)\end{array}$ & $\begin{array}{l}4.04(2.86- \\
5.71)\end{array}$ & .143 \\
\hline $\begin{array}{l}\mathrm{E}_{2} \text { on } \mathrm{Gn} \\
\text { initiation } \\
\text { day }(\mathrm{ng} / \mathrm{ml})\end{array}$ & $\begin{array}{l}24.34(12.88- \\
34.11)\end{array}$ & $\begin{array}{l}26.17(15.44- \\
46.27)\end{array}$ & .305 & $\begin{array}{l}24.48(13.40- \\
34.11)\end{array}$ & $\begin{array}{l}26.10(15.20- \\
46.27)\end{array}$ & .370 \\
\hline \multicolumn{7}{|c|}{ Note: *: Values are significantly different between groups $(P<0.05)$. } \\
\hline
\end{tabular}




\begin{tabular}{|c|c|c|c|c|c|c|}
\hline Variables & Pregnant & Not pregnant & $\begin{array}{l}P \\
\text { value }\end{array}$ & $\begin{array}{l}\text { Ongoing } \\
\text { pregnancy }\end{array}$ & $\begin{array}{l}\text { No ongoing } \\
\text { pregnancy }\end{array}$ & $\begin{array}{l}P \\
\text { value }\end{array}$ \\
\hline $\begin{array}{l}\text { The amount } \\
\text { of } G n(I U)\end{array}$ & $\begin{array}{l}2063(1569- \\
2419)\end{array}$ & $\begin{array}{l}\text { 2025(1588- } \\
2588)\end{array}$ & .915 & $\begin{array}{l}2025(1519- \\
2475)\end{array}$ & $\begin{array}{l}2025(1650- \\
2475)\end{array}$ & .798 \\
\hline $\begin{array}{l}\text { Duration of } \\
\text { stimulation } \\
\text { (d) }\end{array}$ & $9(8-10)$ & $9(8-10)$ & .832 & $9(8-10)$ & $9(8-10)$ & .593 \\
\hline $\begin{array}{l}\text { EM thickness } \\
\text { onHCG } \\
\text { day }(\mathrm{mm})\end{array}$ & $10(9-12)$ & $10(8-11)$ & .060 & $10(9-12)$ & $10(8-11)$ & .076 \\
\hline $\begin{array}{l}\text { P on HCG day } \\
(\mathrm{ng} / \mathrm{ml})\end{array}$ & $\begin{array}{l}0.57(0.40- \\
0.80)\end{array}$ & $\begin{array}{l}0.71(0.48- \\
0.89)\end{array}$ & $.041^{*}$ & $\begin{array}{l}0.58(0.40- \\
0.84)\end{array}$ & $\begin{array}{l}0.67(0.44- \\
0.88)\end{array}$ & .187 \\
\hline $\begin{array}{l}\text { LH on HCG } \\
\text { day }(U / L)\end{array}$ & $\begin{array}{l}2.95(1.32- \\
5.14)\end{array}$ & $\begin{array}{l}3.39(2.07- \\
5.85)\end{array}$ & .075 & $\begin{array}{l}2.90(1.32- \\
5.16)\end{array}$ & $\begin{array}{l}3.33(2.04- \\
5.75)\end{array}$ & .090 \\
\hline $\begin{array}{l}\mathrm{E}_{2} \text { on } \mathrm{HCG} \\
\text { day }(\mathrm{pg} / \mathrm{ml})\end{array}$ & $\begin{array}{l}\text { 1026.5(751.6- } \\
1312.8)\end{array}$ & $\begin{array}{l}\text { 1068.5(614.9- } \\
1539.5)\end{array}$ & .615 & $\begin{array}{l}1037(751.6- \\
1352.5)\end{array}$ & $\begin{array}{l}\text { 1047(614.9- } \\
1503)\end{array}$ & .946 \\
\hline $\begin{array}{l}\text { Oocytes } \\
\text { retrieved (n) }\end{array}$ & $3(2-4)$ & $3(2-4)$ & .316 & $4(4-4)$ & $3(2-4)$ & $<.001^{\star}$ \\
\hline $\begin{array}{l}\text { Mature } \\
\text { oocytes (n) }\end{array}$ & $3(2-4)$ & $3(2-4)$ & .152 & $3(2-4)$ & $3(2-4)$ & .444 \\
\hline $\begin{array}{l}\text { Normal } \\
\text { fertilization } \\
\text { rate }(\%)\end{array}$ & $\begin{array}{l}79.58 \% \\
(191 / 240)\end{array}$ & $\begin{array}{l}72.96 \% \\
(197 / 270)\end{array}$ & .100 & $\begin{array}{l}82.04 \% \\
(169 / 206)\end{array}$ & $\begin{array}{l}71.57 \% \\
(219 / 306)\end{array}$ & $.009^{*}$ \\
\hline $\begin{array}{l}\text { High quality } \\
\text { embryo rate } \\
(\%)\end{array}$ & $\begin{array}{l}72.04 \% \\
(134 / 186)\end{array}$ & $\begin{array}{l}61.75 \% \\
(113 / 183)\end{array}$ & $.047^{*}$ & $\begin{array}{l}70.12 \% \\
(115 / 164)\end{array}$ & $\begin{array}{l}64.39 \% \\
(132 / 205)\end{array}$ & .293 \\
\hline $\begin{array}{l}\text { Embryos } \\
\text { transferred (n) }\end{array}$ & $2(2-2)$ & $2(1-2)$ & $<.001^{*}$ & $2(2-2)$ & $2(1-2)$ & $.001^{*}$ \\
\hline $\begin{array}{l}\text { HQ embryos } \\
\text { transferred (n) }\end{array}$ & $2(1-2)$ & $1(1-2)$ & $.001^{*}$ & $2(1-2)$ & $1(1-2)$ & $.004^{*}$ \\
\hline \multicolumn{7}{|c|}{ Note: *: Values are significantly different between groups $(P<0.05)$. } \\
\hline
\end{tabular}

The clinical outcomes of IVF are summarized in Table 4. Patients who received long-acting GnRH agonist long protocol had a significantly higher clinical pregnancy rate than patients who received GnRH antagonist and mild stimulation protocols $(66.67 \%, 42.17 \%$ and $39.02 \%$, respectively; $P=.024$ and .023$)$. Implantation rates and ongoing pregnancy rates were significantly higher in the group of long-acting GnRH agonist protocol compared with GnRH antagonist protocol (46.15\% and $29.71 \%, 60.00 \%$ and $34.94 \%$, respectively; $P=.035$ and .019$)$, but not significantly higher when compared with mild stimulation protocol (46.15\% and $28.57 \%, 60.00 \%$ and $36.59 \%$, respectively; $P=.079$ and .053$)$. 
Table 4

IVF cycle clinical outcomes between the three groups: univariate and multivariate logistic analysis.

\begin{tabular}{llll}
\hline Outcomes & Group1 & Group2 & Group 3 \\
\hline Number of cycles & 180 & 122 & 36 \\
\hline Clinical pregnancy rate $(\%)$ & $39.02 \%(16 / 41)$ & $42.17 \%(35 / 83)$ & $66.67 \%(20 / 30)$ \\
\hline Univariate $(\mathrm{OR}(95 \% \mathrm{Cl}), P)$ & $\begin{array}{l}0.32(0.12-0.84), \\
0.023^{\star}\end{array}$ & $0.36(0.15-0.86), 0.024^{\star}$ & 1 \\
\hline Multivariate $(\mathrm{OR}(95 \% \mathrm{Cl}), P)$ & $\begin{array}{l}0.14(0.03-0.60), \\
0.010^{\star}\end{array}$ & $0.18(0.05-0.57), 0.005^{\star}$ & 1 \\
\hline Implantation rate $(\%)$ & $28.57 \%(18 / 63)$ & $29.71 \%(41 / 138)$ & $46.15 \%(24 / 52)$ \\
\hline Univariate $(\mathrm{OR}(95 \% \mathrm{Cl}), P)$ & $0.50(0.23-1.08), 0.079$ & $0.49(0.26-0.95), 0.035^{\star}$ & 1 \\
\hline Multivariate $(\mathrm{OR}(95 \% \mathrm{Cl}), P)$ & $\begin{array}{l}0.34(0.12-0.95), \\
0.041^{*}\end{array}$ & $0.41(0.18-0.89), 0.025^{\star}$ & 1 \\
\hline $\begin{array}{l}\text { Ongoing pregnancy rate } \\
\text { (\%) }\end{array}$ & $36.59 \%(15 / 41)$ & $34.94 \%(29 / 83)$ & $60.00 \%(18 / 30)$ \\
\hline Univariate $(\mathrm{OR}(95 \% \mathrm{Cl}), P)$ & $0.38(0.14-1.00), 0.053$ & $0.36(0.15-0.84), 0.019^{\star}$ & 1 \\
\hline Multivariatec $(\mathrm{OR}(95 \% \mathrm{Cl}), P)$ & $\begin{array}{l}0.20(0.05-0.74), \\
0.018^{\star}\end{array}$ & $0.21(0.07-0.59), 0.004^{\star}$ & 1 \\
\hline
\end{tabular}

Note: *: Values are significantly different between groups $(P<0.05)$.

a. Adjusted for duration of infertility, AFC, amount of gonadotrophins, $E_{2}$ on HCG day, number of embryos transferred and number of high quality embryos transferred.

b: Adjusted for duration of infertility, AFC, amount of gonadotrophins, P on HCG day and number of high quality embryos transferred.

c: Adjusted for duration of infertility, amount of gonadotrophins and number of embryos transferred.

However, clinical pregnancy rates, implantation rates and ongoing pregnancy rates were also significantly higher among the women who received long-acting GnRH agonist compared with the other two groups after adjusting for potential confounding factors (duration of infertility, AFC, amount of gonadotrophins, $P$ and $E_{2}$ on HCG day, and the number of embryo and high quality embryo transferred $)(P=.010, .041$ and $.018, P=.005, .025$ and .004 , respectively).

To explore the mechanism of long-acting GnRH agonist long protocol in follicular phase improving IVF outcomes of young patients with DOR, we have assessed the morphology and coverage of pinopode and expression of HOXA10 between three groups. The result showed that there were significantly differences in the proportion of pinopodes with different morphologies and pinopode coverage between the three 
groups. Long-acting GnRH agonist long protocol in follicular phase could improve the development of pinopode, and the pinopode coverage was higher compared with the other groups (Fig. 3). In addition, the mRNA of HOXA10 was significantly higher among these patients who received long-acting GnRH agonist compared with mild stimulation and GnRH antagonist protocols (Fig. 4).

\section{Discussion}

Here, for the first time, we evaluated the effect of long-acting GnRH agonist long protocol in follicular phase on IVF cycle outcomes in young patients with DOR compared with conventional protocols. Longacting $\mathrm{GnRH}$ agonist long protocol in follicular phase is a novel ovarian stimulation protocol. Recently, some studies found that this protocol may improve endometrial receptivity and clinical outcomes of IVF [16-19].

In this paper, we showed that young DOR patients who received long-acting GnRH agonist long protocol had significantly higher endometrial thickness, clinical pregnancy rates, implantation rates and ongoing pregnancy rates when compared with conventional protocols. Moreover, they had significantly lower embryo transfer cancellation rates and abnormal endometrium rates. However, the duration of stimulation and dose of gonadotrophins were significantly higher among women who received longacting $\mathrm{GnRH}$ agonist. These results suggest that long-acting $\mathrm{GnRH}$ agonist long protocol in follicular phase may further suppress ovarian response, increase endometrial thickness, reduce cycle cancellation rate and enhance clinical pregnancy rate, implantation rate and ongoing pregnancy rate for young DOR patients. Therefore, we believe that long-acting $\mathrm{GnRH}$ agonist long protocol can improve the clinical outcomes of IVF for young DOR patients.

To explore the mechanism of long-acting GnRH agonist improving IVF outcomes of young patients with DOR, we have assessed the morphology and coverage of pinopode and expression of HOXA10 between three groups. We found that long-acting $\mathrm{GnRH}$ agonist could improve the development of pinopodes and expression of HOXA10. As we all know, pinopode and HOXA10 are the important markers of endometrial receptivity. Therefore, we thought that long-acting $\mathrm{GnRH}$ agonist could improve endometrial receptivity.

During the past few decades, GnRH agonist has been widely used during controlled ovarian stimulation in assisted reproductive treatment cycles to avoid premature endogenous peak of luteinizing hormone [24]. There are two types of $\mathrm{GnRH}$ agonist administration to lead to hypophysis desensitization, including daily $\mathrm{GnRH}$ agonist low doses and depot long-acting doses. In addition, GnRH agonist administration is started in follicular phase, and another in the middle of the previous luteal phase.

Many studies compared the effect of long-acting and short-acting GnRH agonist on IVF cycle outcomes [25-29]. Some studies indicated that long-acting GnRH agonist did not improve the outcomes of IVF, as compared with short-acting GnRH agonist. However, the use of depot $\mathrm{GnRH}$ agonist increased the number of gonadotrophins and the duration of the ovarian stimulation. We have carefully reviewed these papers, and found that the pituitary desensitization time of long-acting and short-acting GnRH agonist were same and short from 14 to 21 days before ovarian stimulation. Recently, the novel ovarian stimulation protocol, 
long-acting $\mathrm{GnRH}$ agonist long protocol in follicular phase, was proposed in IVF cycle [14-19]. In this protocol, the duration of pituitary desensitization is extended to more than one month before ovarian stimulation. Several studies have demonstrated that this prolonged pituitary down-regulation protocol can significantly improve IVF cycle outcomes compared with other protocols including short-acting $\mathrm{GnRH}$ agonist long protocol [16-19]. Moreover, administration of long-acting GnRH agonist for 2-6 months preIVF cycle in this endometriosis patient population resulted in significantly higher clinical pregnancy rates, ongoing pregnancy rates and live birth rates [30-32]. In this paper, we also found that the incidence of endometriosis was highest in the long-acting $\mathrm{GnRH}$ agonist group, but the clinical pregnancy rates, implantation rate and ongoing pregnancy rates were significantly higher than the other groups. These results imply that prolonged pituitary down-regulation by using long-acting $\mathrm{GnRH}$ agonist might improve the outcomes of IVF.

As we all know, DOR patients have few antral follicle counts. Long-acting GnRH agonist may result in excessive ovarian suppression. Therefore, the conventional ovarian stimulation protocols are mainly mild ovarian stimulation and $\mathrm{GnRH}$ antagonist protocols for DOR patients $[7,8,10,13]$. However, in our present study, our data indicate that long-acting $\mathrm{GnRH}$ agonist could improve endometrial thickness and clinical outcomes of IVF for young DOR patients. In addition, our results showed that this protocol could increase the amount of gonadotropins and the duration of ovarian stimulation, but did not affect embryo quality. This is in accordance with previous works [18]. Furthermore, long-acting GnRH agonist long protocol is much more comfortable for patients, requiring only a single depot dose of $\mathrm{GnRH}$ agonist in follicular phase.

Though this long-acting GnRH agonist protocol may lead to excessive ovarian suppression, it can increase endometrial thickness, reduce cycle cancellation rate and improve clinical outcomes of IVF for young DOR patients. However, the mechanism of action of long-acting GnRH agonist long protocol on improving IVF outcomes has not been elucidated. Many researchers believe that long-acting $\mathrm{GnRH}$ agonist may improve endometrial receptivity [16-19]. Firstly, the GnRH agonist had a direct effect on endometrium by regulating the expression of the enzymes and cytokines to improve endometrial receptivity [32-37]. Integrin av $\beta 3$ plays an important role during embryo implantation. Absent or aberrant endometrial expression of this protein in women with infertility had been reported. Long-acting $\mathrm{GnRH}$ agonist could normalize the aberrant $\beta 3$ integrin expression [32,34]. Secondly, after a natural or induced period of amenorrhea before embryo transfer, the uterine may restore full function to the steroid-sensitive systems. This may result in the restoration of uterine capacity for embryo implantation and improvement in clinical pregnancy rate $[38,39]$. Amenorrhea induced by pituitary down-regulation with long-acting $\mathrm{GnRH}$ agonist might also have the same effect. A period of pituitary down-regulation might increase endometrial receptivity, as a favorable factor for infertile patients [40]. Finally, the thickness of endometrium has been used as a marker of adequate endometrial receptivity. The pregnancy rate increases as endometrial thickness, independent of the number and quality of embryos transferred [4145]. Long-acting GnRH agonist could increase the endometrial thickness to improve embryo implantation. Therefore, we believe that long-acting $\mathrm{GnRH}$ agonist long protocol in follicular phase might result in 
excessive ovarian suppression and a period of amenorrhea to improve the endometrial thickness, endometrial receptivity and clinical outcomes of IVF for young DOR patients.

As a retrospective study, some limitations must be also considered. Firstly, a retrospective cross-sectional study has many confounding factors. Therefore, we have adjusted the potential confounding factors to provide reassurance of the validity of our findings in this study. Secondly, the sample size of our study was relatively small, especially in the long-acting GnRH agonist group. However, our study was a single centre study allowing for uniformity of treatment protocols. In future, an ideal large randomized controlled trial should be performed.

Despite these limitations, this study still had several strengths. The primary strength is that this is the first publication of using long-acting GnRH agonist in young DOR patients. In addition, the population was homogeneous. They were all of Chinese origin and were treated in a single centre, which allows for standardization of ovarian stimulation protocols, laboratory and embryo transfer techniques.

\section{Conclusions}

In summary, this is the first report that long-acting GnRH agonist was administrated for young DOR patients. We demonstrated for the first time that long-acting $\mathrm{GnRH}$ agonist long protocol in follicular phase could improve IVF outcomes for young DOR patients, as compared with conventional protocols. In addition, we found that long-acting $\mathrm{GnRH}$ agonist may not affect embryo quality but improve endometrial receptivity. These encouraging results from this retrospective study need to be confirmed by a large randomized controlled trial in future.

\section{Abbreviations}

DOR

diminished ovarian reserve; GnRH:gonadotrophin-releasing hormone; IVF:in vitro fertilization; AMH:antiMullerian hormone; ART:assisted reproductive technologies; AFC:antral follicle count; ICSI:intracytoplasmic sperm injection; BMI:body mass index.

\section{Declarations}

\section{Funding}

This work was supported by National Natural Science Foundation of China (81601276) and Yantai Science and Technology Project (2020YD006).

\section{Acknowledgments}

Thanks for the outstanding support provided by the whole staff of our reproductive medicine center.

\section{Availability of data and meterials}


The primary data for this study is available from patient medical records.

\section{Authors' contributions}

XM Liu, HC Bao and QL Qu were involved in the study design, drafted and revision of the manuscript. ZY Guo, WS Li and LQ Zhang analyzed data. HS Zhao and Z Ma were involved in the critical revision of the manuscript. All authors approved the final version for submission.

\section{Ethics approval and consent to participate}

The study was approved by the Ethical Committee of Yantai Yuhuangding Hospital.

\section{Consent for publication}

Not applicable.

\section{Competing interests}

The authors declare that they have no competing interests.

\section{Author details}

${ }^{1}$ Reproductive Medicine Center, Yantai Yuhuangding Hospital, Affiliated Hospital of Qingdao University, Yantai, Shandong, China. ${ }^{2}$ Department of pharmacy, Yantai Yuhuangding Hospital, Affiliated Hospital of Qingdao University, Yantai, Shandong, China.

\section{References}

1. Broekmans FJ, Soules MR, Fauser BC. Ovarian aging: mechanisms and clinical consequences. Endocr Rev. 2009;30:465-93.

2. Scott RT, Leonardi MR, Hofmann GE, Illions EH, Neal GS, Navot D. A prospective evaluation of clomiphene citrate challenge test screening of the general infertility population. Obstet Gynecol. 1993;82:539-44.

3. Greene AD, Patounakis G, Segars JH. Genetic associations with diminished ovarian reserve: a systematic review of the literature. J Assist Reprod Genet. 2014;31:935-46.

4. Practice Committee of the American Society for Reproductive Medicine. Testing and interpreting measures of ovarian reserve: A committee opinion. Fertil Steril. 2012;98:1407-15.

5. Broekmans FJ, Kwee J, Hendriks DJ, Mol BW, Lambalk CB. A systematic review of tests predicting ovarian reserve and IVF outcome. Hum Reprod Update. 2006;12:685-718.

6. Levi AJ, Raynault MF, Bergh PA, Drews MR, Miller BT, Scott RT. Reproductive outcome in patients with diminished ovarian reserve. Fertil Steril. 2001;76:666-69. 
7. Lin MH, Wu FS, Hwu YM, Lee RK, Li RS, Li SH. Dual trigger with gonadotropin releasing hormone agonist and human chorionic gonadotropin significantly improves live birth rate for women with diminished ovarian reserve. Reprod Biol Endocrinol. 2019;17:7.

8. Huang MC, Tzeng SL, Lee $\mathrm{Cl}$, Chen $\mathrm{HH}$, Huang CC, Lee TH, et al. GnRH agonist long protocol versus $\mathrm{GnRH}$ antagonist protocol for various aged patients with diminished ovarian reserve: A retrospective study. PloS One. 2018;13:e0207081.

9. Xu Y, Nisenblat V, Lu C, Li R, Qiao J, Zhen X, et al. Pretreatment with coenzyme Q10 improves ovarian response and embryo quality in low-prognosis young women with decreased ovarian reserve: a randomized controlled trial. Reprod Biol Endocrinol. 2018;16:29.

10. Olgan S, Humaidan P. GnRH antagonist and letrozole co-treatment in diminished ovarian reserve patients: a proof-of-concept study. Reprod Biol. 2017;17:105-10.

11. Li J, Yuan H, Chen Y, Wu H, Wu H, Li L. A meta-analysis of dehydroepiandrosterone supplementation among women with diminished ovarian reserve undergoing in vitro fertilization or intracytoplasmic sperm injection. Int J Gynaecol Obstet. 2015;131:240-5.

12. Grisendi V, Mastellari E, La Marca A. Ovarian Reserve Markers to Identify Poor Responders in the Context of Poseidon Classification. Front Endocrinol (Lausanne). 2019;10:281.

13. Youssef MA, van Wely M, Al-Inany H, Madani T, Jahangiri N, Khodabakhshi S, et al. A mild ovarian stimulation strategy in women with poor ovarian reserve undergoing IVF: a multicenter randomized non-inferiority trial. Hum Reprod. 2017;32:112-8.

14. Kong H, Bu Z, Guo Y, Wang F, Shi H, Hu L, et al. Efficacy and Safety of In Vitro Fertilization (IVF)/Intracytoplasmic Sperm Injection (ICSI) Among Patients with Endometriosis After a Shortened Protocol of Long-Term Pituitary Downregulation. Med Sci Monit. 2019;25:4377-83.

15. Kong H, Hu L, Nie L, Yu X, Dai W, Li J, et al. A multi-center, randomized controlled clinical trial of the application of a shortened protocol of long-acting Triptorelin down-regulated prior to IVF/ICSI among patients with endometriosis: A protocol. Reprod Health. 2018;15:213.

16. Song J, Sun X, Qian K. Endometrial but not Ovarian Response is Associated With Clinical Outcomes and can be Improved by Prolonged Pituitary Downregulation in Patients With Thin and Medium Endometrium. Reprod Sci. 2018; Dec 3:1933719118816835. doi: 10.1177/1933719118816835.

17. Chen X, Feng SX, Guo PP, He YX, Liu YD, Ye DS, et al. Does Lower Dose of Long-acting Triptorelin Maintain Pituitary Suppression and Produce Good Live Birth Rate in Long Down-regulation Protocol for In-vitro Fertilization. J Huangzhong Univ Sci Technolog Med Sci. 2016;36:215-20.

18. Mao GH, Feng Z, He Y, Huang YR. Comparisons of the effects of long-acting and short-acting GnRH agonists on embryo quality, endometrial thickness and pregnancy rate in human in vitro fertilization. Arch Med Sci. 2014;10:161-6.

19. Ren J, Sha A, Han D, Li P, Geng J, Ma C. Does prolonged pituitary down-regulation with gonadotropinreleasing hormone agonist improve the live-birth rate in in vitro fertilization treatment? Fertil Steril. 2014;102:75-81. 
20. van der Houwen LE, Mijatovic V, Leemhuis E, Schats R, Heymans MW, Lambalk CB, et al. Efficacy and safety of IVF/ICSI in patients with severe endometriosis after long-term pituitary down-regulation. Reprod Biomed Online. 2014;28:39-46.

21. Sallam HN, Garcia-Velasco JA, Dias S, Arici A. Long-term pituitary down-regulation before in vitro fertilization (IVF) for women with endometriosis. Cochrane Database Syst Rev. 2006; (1):CD004635.

22. Cohen J, Chabbert-Buffet N, Darai E. Diminished ovarian reserve, premature ovarian failure, poor ovarian responder-a plea for universal definitions. J Assist Reprod Genet. 2015;32:1709-12.

23. Balaban B, Brison D, Calderon G, Catt J, Conaghan J, Cowan L, et al. The Istanbul consensus workshop on embryo assessment: proceedings of an expert meeting. Hum Reprod. 2011; 26: 127083.

24. Caspi E, Ron-El R, Golan A, Nachum H, Herman A, Soffer Y, et al. Results of in vitro fertilization and embryo transfer by combined long-acting gonadotropin-releasing hormone analog D-Trp-6-luteinizing hormone-releasing hormone and gonadotropins. Fertil Steril. 1989;51:95-9.

25. Albuquerque LE, Saconato H, Maciel MC. Depot versus daily administration of gonadotrophin releasing hormone agonist protocols for pituitary desensitization in assisted reproduction cycles. Cochrane Database Syst Rev. 2005; (1):CD002808.

26. Isikoglu M, Ozdem S, Berkkanoglu M, Jamal H, Senturk Z, Ozgur K. Single-dose depot leuprolide is as efficient as daily short-acting leuprolide in ICSI cycles. Hum Reprod. 2007;22:1657-61.

27. Duan L, Bao S, Li K, Teng X, Hong L, Zhao X. Comparing the long-acting and short-acting forms of gonadotropin-releasing hormone agonists in the long protocol of IVF/ICSI Cycles: A retrospective study. J Obstet Gynaecol Res. 2017;43:1037-42.

28. Dada T, Salha O, Baillie HS, Sharma V. A comparison of three gonadotrophin-releasing hormone analogues in an in-vitro fertilization programme: a prospective randomized study. Hum Reprod. 1999;14:288-93.

29. Hsieh YY, Chang CC, Tsai HD. Comparisons of different dosages of gonadotropin-releasing hormone $(\mathrm{GnRH})$ antagonist, short-acting form and single, half-dose, long-acting form of GnRH agonist during controlled ovarian hyperstimulation and in vitro fertilization. Taiwan J Obstet Gynecol. 2008;47:6674.

30. Surrey ES, Silverberg KM, Surrey MW, Schoolcraft WB. Effect of prolonged gonadotropin-releasing hormone agonist therapy on the outcome of in vitro fertilization-embryo transfer in patients with endometriosis. Fertil Steril. 2002;78:699-704.

31. Sallam HN, Garcia-Velasco JA, Dias S, Arici A. Long term pituitary down-regulation before in vitro fertilization (IVF) for women with endometriosis. Cochrane Database Syst Rev. 2006; (1): CD004635.

32. Surrey ES, Minjarez DA, Schoolcraft WB. The incidence of aberrant endometrial av $\beta 3$ vitronectin expression in a high-risk fertility population: could prolonged $\mathrm{GnRH}$ agonist therapy play a role? J Assist Reprod Genet. 2007;24:553-6.

33. Wang JH, Zhou FZ, Dong MY, Wu RJ, Qian YL. Prolonged gonadotropin-releasing hormone agonist therapy reduced expression of nitric oxide synthase in the endometrium of women with 
endometriosis and infertility. Fertil Steril. 2006;85:1037-44.

34. Lessey B. Medical management of endometriosis and infertility. Fertil Steril. 2000;73:1089-96.

35. Khan KN, Kitajima M, Hiraki K, Fujishita A, Sekine I, Ishimaru T, et al. Changes in tissue inflammation, angiogenesis and apoptosis in endometriosis, adenomyosis and uterine myoma after $\mathrm{GnRH}$ agonist therapy. Hum Reprod. 2010;25:642-53.

36. Sakamoto Y, Harada T, Horie S, Iba Y, Taniguchi F, Yoshida S, et al. Tumor necrosis factor-alphainduced interleukin-8 (IL-8) expression in endometriotic stromal cells, probably through nuclear factor-kappa B activation: gonadotropin-releasing hormone agonist treatment reduced IL-8 expression. J Clin Endocrinol Metab. 2003;88:730-5.

37. Kim YA, Kim MR, Lee JH, Kim JY, Hwang KJ, Kim HS, et al. Gonadotropin releasing hormone agonist reduces aromatase cytochrome $\mathrm{P} 450$ and cyclooxygenase- 2 in ovarian endometrioma and eutopic endometrium of patients with endometriosis. Gynecol Obstet Invest. 2009;68:73-81.

38. Edwards RG. Clinical approaches to increasing uterine receptivity during human implantation. Hum Reprod. 1995;10(Suppl 2):60-6.

39. Edwards RG, Morcos S, Macnamee M, Balmaceda JP, Walters DE, Asch R. High fecundity of amenorrhoeic women in embryo-transfer programmes. Lancet. 1991;338:292-4.

40. Flamigni C, Borini A. Counselling post-menopausal women for donor in-vitro fertilization and hormone replacement therapy. Hum Reprod. 1995;10:1237-41.

41. Yang W, Zhang T, Li Z, Ren X, Huang B, Zhu G, et al. Combined analysis of endometrial thickness and pattern in predicting clinical outcomes of frozen embryo transfer cycles with morphological goodquality blastocyst: A retrospective cohort study. Medicine. 2018;97:e9577.

42. Zhao J, Zhang Q, Wang Y, Li Y. Endometrial pattern, thickness and growth in predicting pregnancy outcome following 3319 IVF cycle. Reprod Biomed Online. 2014;29:291-8.

43. Richter KS, Bugge KR, Bromer JG, Levy MJ. Relationship between endometrial thickness and embryo implantation, based on 1,294 cycles of in vitro fertilization with transfer of two blastocyst-stage embryos. Fertil Steril. 2007;87:53-9.

44. Al-Ghamdi A, Coskun S, Al-Hassan S, Al-Rejjal R, Awartani K. The correlation between endometrial thickness and outcome of in vitro fertilization and embryo transfer (IVF-ET) outcome. Reprod Biol Endocrinol. 2008;6:37.

45. Chen SL, Wu FR, Luo C, Chen X, Shi XY, Zheng HY, et al. Combined analysis of endometrial thickness and pattern in predicting outcome of in vitro fertilization and embryo transfer: a retrospective cohort study. Reprod Biol Endocrinol. 2010;8:30.

\section{Figures}


Long-acting $\mathrm{GnRH}$-a long protocol
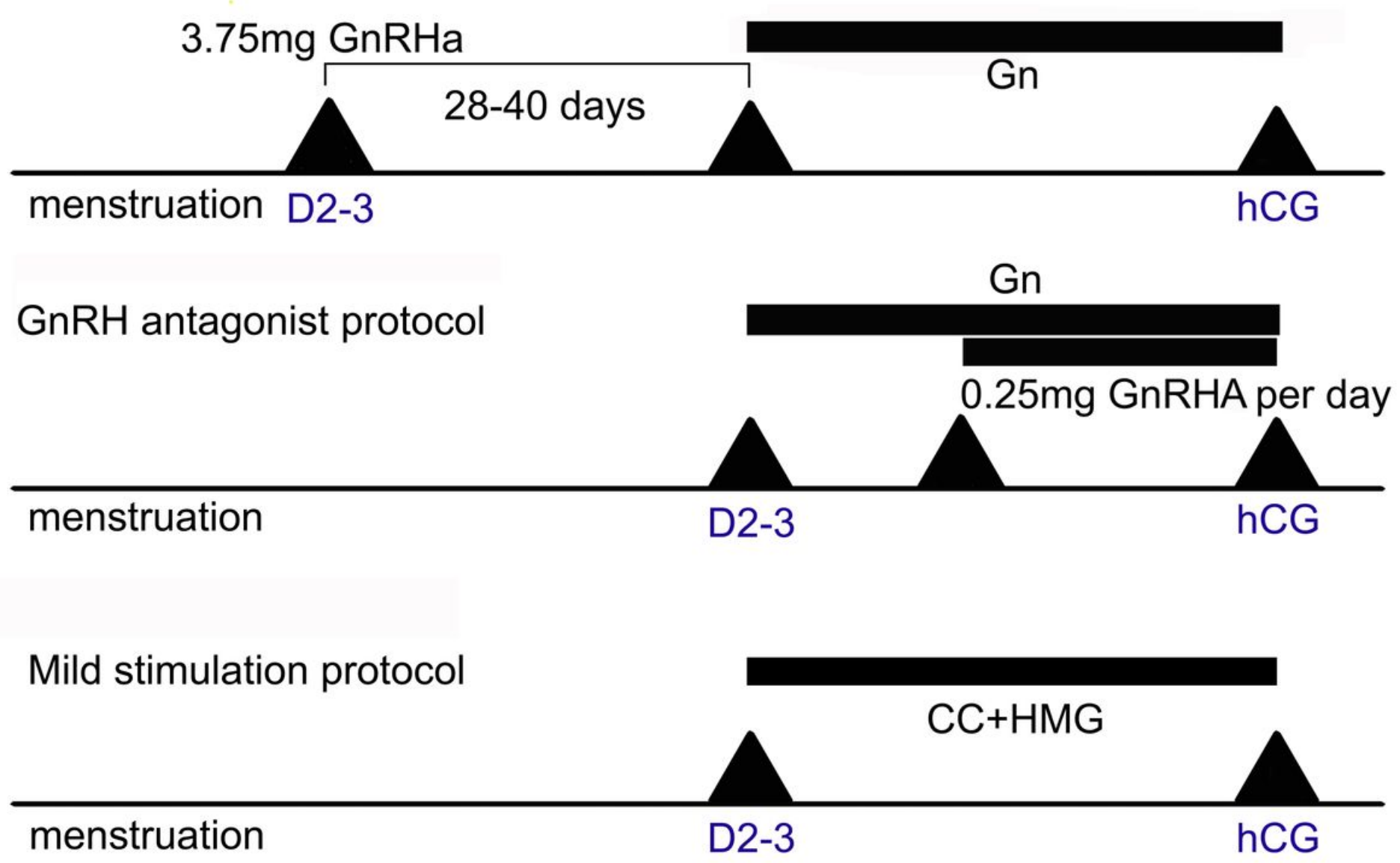

Figure 1

The protocols of ovarian stimulation. 


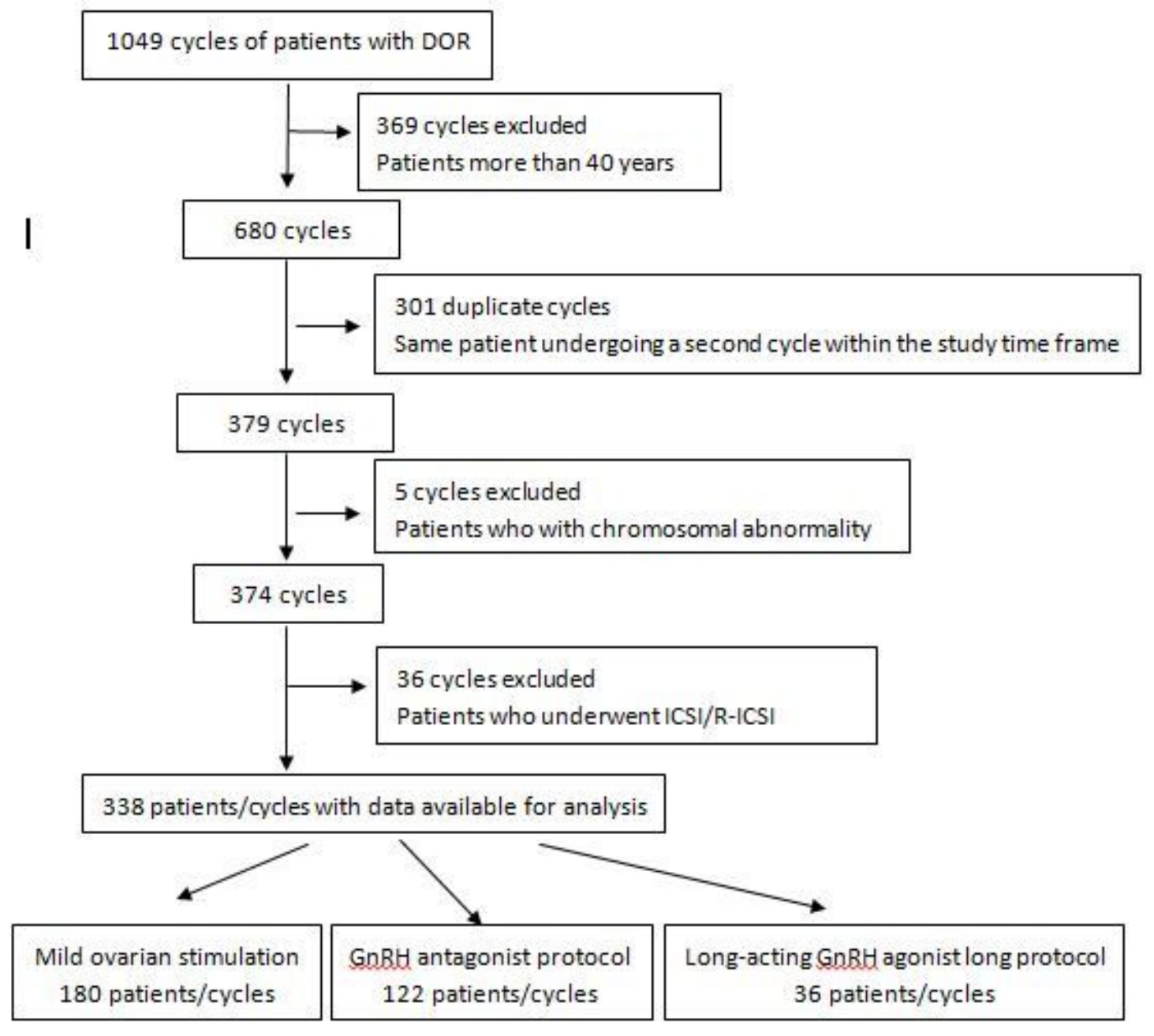

Figure 2

Flow chart of the patient selection process.

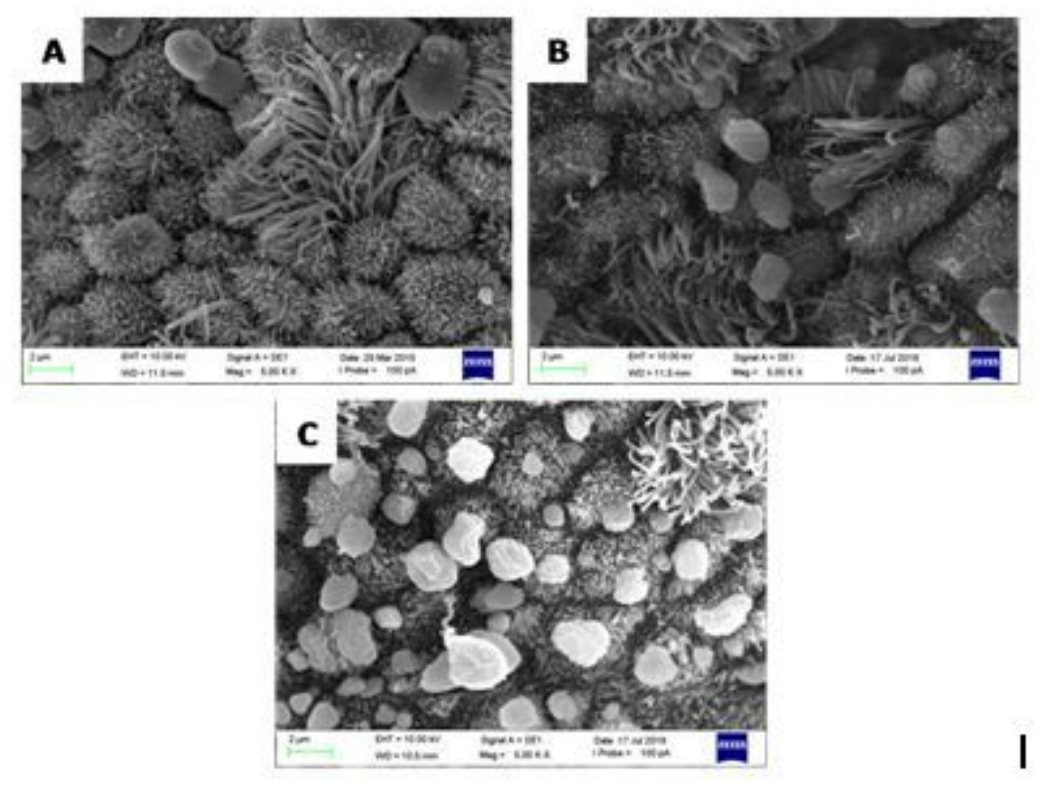

Figure 3 
Representative scanning electron micrographs of pinopode coverage in the endometrial epithelium on Day after oocyte retrieval. A: mild ovarian stimulation protocol, B: GnRH antagonist protocol and C: longacting $\mathrm{GnRH}$ agonist long protocol in follicular phase.

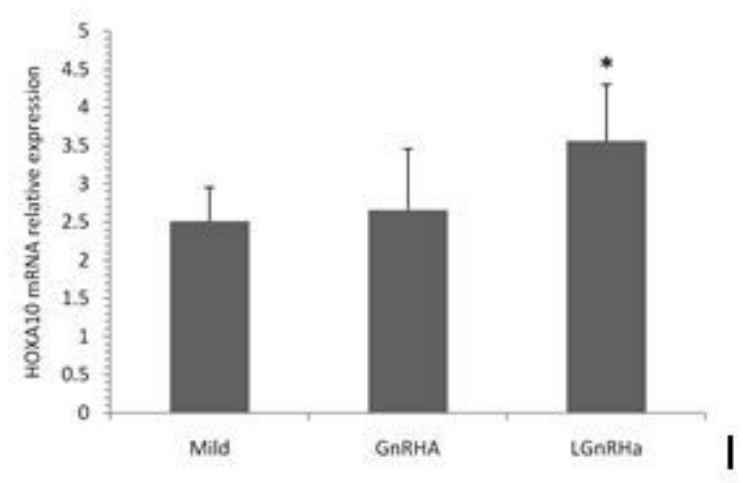

Figure 4

HOXA10 mRNA in the endometrial epithelium on Day after oocyte retrieval. Mild: mild ovarian stimulation protocol, GnRHA: GnRH antagonist protocol and LGnRHa: long-acting GnRH agonist long protocol in follicular phase. Statistical significance denoted by *P囚.05. 